\title{
BMJ Open Initial implementation of PrEP in Zambia: health policy development and service delivery scale-up
}

Cassidy W. Claassen (D) , ${ }^{1,2,3}$ Daliso Mumba, ${ }^{4,5}$ Mwansa Njelesani, ${ }^{6}$
Derrick Nyimbili, ${ }^{7}$ Linah K Mwango, ${ }^{8}$ Mundia Mwitumwa, ${ }^{2,3}$ Ellen Mubanga, ${ }^{4}$
Lloyd B. Mulenga, ${ }^{2,3,5}$ Tina Chisenga, ${ }^{5}$ Brooke E. Nichols ${ }_{9,10}$
Cheryl Hendrickson (D) , ${ }^{10}$ Lastone Chitembo, ${ }^{11}$ Jackson Okuku, ${ }^{12}$ Heidi O'Bra $^{13}$

\section{ABSTRACT}

Mumba D, Njelesani M, et al. Initial implementation of PrEP in Zambia: health policy development and service delivery scale-up. BMJ Open 2021;11:e047017. doi:10.1136/ bmjopen-2020-047017

- Prepublication history for this paper is available online. To view these files, please visit the journal online (http://dx.doi. org/10.1136/bmjopen-2020047017).

Received 07 December 2020 Accepted 10 June 2021

Check for updates

(C) Author(s) (or their employer(s)) 2021. Re-use permitted under CC BY-NC. No commercial re-use. See rights and permissions. Published by BMJ.

For numbered affiliations see end of article.

Correspondence to Dr Cassidy W. Claassen; cclaassen@ihv.umaryland.edu respectively.
Introduction Daily pre-exposure prophylaxis (PrEP) for HIV prevention is highly effective, but not yet widely deployed in sub-Saharan Africa. We describe how Zambia developed PrEP health policy and then successfully implemented national PrEP service delivery.

Policy development Zambia introduced PrEP as a key strategy for HIV prevention in 2016, and established a National PrEP Task Force to lead policy advocacy and development. The Task Force was composed of government representatives, regulatory agencies, international donors, implementation partners and civil society organisations. Following an implementation pilot, PrEP was rolled out nationally using risk-based criteria alongside a national HIV prevention campaign.

National Scale-up In the first year of implementation, ending September 2018, 3626 persons initiated PrEP. By September 2019, the number of people starting PrEP increased by over sixfold to 23327 persons at 728 sites across all ten Zambian provinces. In the first 2 years, 26953 clients initiated PrEP in Zambia, of whom 31\% were from key and priority populations. Continuation remains low at $25 \%$ and $11 \%$ at 6 and 12 months,

Lessons learnt Risk-based criteria for PrEP ensures access to those most in need of HIV prevention. Healthcare worker training in PrEP service delivery and health needs of key and priority populations is crucial. PrEP expansion into primary healthcare clinics and community education is required to reach full potential. Additional work is needed to understand and address low PrEP continuation. Finally, a task force of key stakeholders can rapidly develop and implement health policy, which may serve as a model for countries seeking to implement PrEP.

\section{INTRODUCTION}

Daily pre-exposure prophylaxis (PrEP) with tenofovir disoproxil fumarate and emtricitabine for the prevention of HIV infection is a highly effective prevention tool, recommended for persons at substantial risk of HIV infection by WHO since 2015. ${ }^{1}$ PrEP effectiveness is highly correlated to adherence, ${ }^{2-6}$ with studies showing that PrEP is most effective
Strengths and limitations of this study

- This study documents how the Zambian preexposure prophylaxis (PrEP) Task Force, consisting of health professionals from government, donors and implementing partners, rapidly drafted and implemented national PrEP health policy.

- This study describes the national implementation and scale up of PrEP to $>700$ health facilities and nearly 27000 clients in 2 years.

- PrEP service delivery is examined by key population type as well as mapped to population density and HIV prevalence.

- A limitation of this study is the use of aggregate data; thus it does not include patient-level data for better understanding of PrEP uptake and continuation.

- The Zambia PrEP health policy development model may be useful to other countries seeking to implement PrEP.

when adherence is $>70 \% 0 .{ }^{7} \mathrm{PrEP}$ has the potential to limit HIV acquisition among at-risk populations, including those who may not be able to negotiate condom usage or who engage in high-risk activities. PrEP can be taken discreetly, it offers high rates of protection, and does not require negotiation with partners, unlike barrier methods and microbicide gels. ${ }^{89} \mathrm{PrEP}$ is also associated with few safety risks, and there is limited evidence of behavioural risk compensation. ${ }^{7}$ Despite these promising results, PrEP roll-out has been slow in sub-Saharan Africa with only a handful of countries implementing large scale national PrEP programmes, including South Africa, Kenya, Uganda, Zimbabwe and Zambia. ${ }^{10}$

Initial PrEP demonstration implementation projects have shown high uptake among serodiscordant couples (SDCs), ${ }^{11} 12$ adolescent girls and young women (AGYW), ${ }^{13} 14$ female sex workers (FSWs) ${ }^{15-17}$ and men who have sex with men (MSM). ${ }^{18} 19$ Interest in, and 
uptake of, PrEP is high but PrEP continuation remains a challenge in many groups, particularly AGYW. Successful national PrEP programmes in Africa have invested in creating PrEP awareness and demand via mass media and community health talks, have streamlined PrEP delivery via not only ART clinics but also drop-in centres for key populations (KPs) and DREAMS (Determined, Resilient, Empowered, AIDS-free, Mentored, Safe) centres for AGYW, and have built PrEP capacity among healthcare workers (HCWs) via trainings and mentorship. Challenges in PrEP programming persist, including the need to improve PrEP access, streamline PrEP delivery models, increase HCWs trained in PrEP and finally increase PrEP continuation. $^{10}$

Zambia's HIV epidemic is generalised, with a persistently high prevalence of $12.0 \%$ and annual incidence of $0.61 \%$ among adults $15-59$ years old. ${ }^{20}$ Concentrated pockets of high HIV transmission disproportionately affect populations who face substantial psychosocial and structural barriers to accessing health services and continue to drive epidemic transmission. These include SDCs; AGYW; FSW and their clients; $\mathrm{MSM}^{21}$; transgendered (TG) individuals $^{22}$ and high-risk men, particularly those under 30 , engaged in migrant work (eg, truck drivers, ${ }^{23}$ cane cutters $^{24}$ or in prison). ${ }^{2526}$ These groups have a higher HIV prevalence compared with the general population, and would benefit the most from HIV prevention methods, including biomedical interventions such as PrEP.

Beginning in 2016, Zambia introduced PrEP as a component of combination HIV prevention services, which also includes counselling, family planning, voluntary male medical circumcision and condom distribution. In this paper, we present a model of integrated health policy and technical service delivery accomplished by

\section{WHO Guidelines on when to start \\ antiretroviral therapy and PrEP for HIV}

Supports PrEP for persons at risk of HIV

Sept. 2015

\section{Zambia National PrEP Task Force}

Develops health policy guidance

2017-2018 government agencies, donors, and implementing partners for the roll-out of PrEP in Zambia. We also report on the current status, challenges faced, and lessons learnt from PrEP policy and service delivery implementation in Zambia.

\section{PrEP policy and implementation}

Development of PreP health policy and implementation guidelines in Zambia

In 2016, the Zambian Ministry of Health (MOH) adopted the 2015 WHO guidelines ${ }^{1}$ recommending daily oral PrEP for people at substantial risk of HIV infection, defined as HIV incidence greater than 3 per 100 personyears (see figure 1 for a timeline of PrEP policy and implementation). PrEP was introduced in the 2016 Zambia Consolidated Guidelines for Treatment and Prevention of HIV Infection ${ }^{27}$ and further expanded in the 2018 version. ${ }^{28}$ In September 2017, the National PrEP Task Force was established, comprising key personnel from $\mathrm{MOH}$, National HIV/AIDS/STI/TB Council, WHO, UNAIDS, US President's Emergency Plan for AIDS Relief (PEPFAR), US Agency for International Development (USAID), US Centers for Disease Control and Prevention (CDC), PEPFAR implementing partners (IPs) and civil society organisations (CSOs). The Task Force's mandate was to lead policy advocacy and formulate a national PrEP implementation framework to guide PrEP service delivery. The Task Force created three sub-committees: (1) medical/technical service delivery and monitoring and evaluation (M\&E); (2) logistics and supply chain and (3) social behavioural change communication and policy advocacy. Within each subcommittee, technical experts reviewed the latest evidence and guidelines for the larger Task Force (table 1, next page). Additionally, the Task

\section{Zambia}

Consolidated Guidelines

for Treatment and

Prevention of HIV Infection

Supports PrEP based on risk criteria

Dec. 2017

\section{National roll-out and scale-up of PrEP}

PrEP service delivery supported at 728 facilities

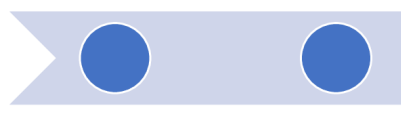

Dec. 2016

2016 Zambia

Consolidated Guidelines

for Treatment and

Prevention of HIV Infection

Supports PrEP for SDCS and high-risk persons
2017-2019

Pilot PrEP Implementation

UMB and UTH conduct

first pilots at 3 sites in Lusaka and Livingstone
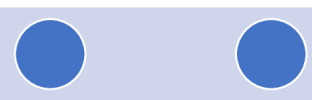

July 2018

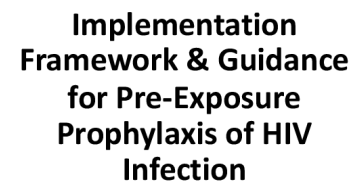

Provides guidance on PrEP service delivery

Figure 1 Timeline of PrEP policy and implementation in Zambia. PrEP, pre-exposure prophylaxis; SDC, serodiscordant couples; UMB, University of Maryland Baltimore; UTH, University Teaching Hospital. 


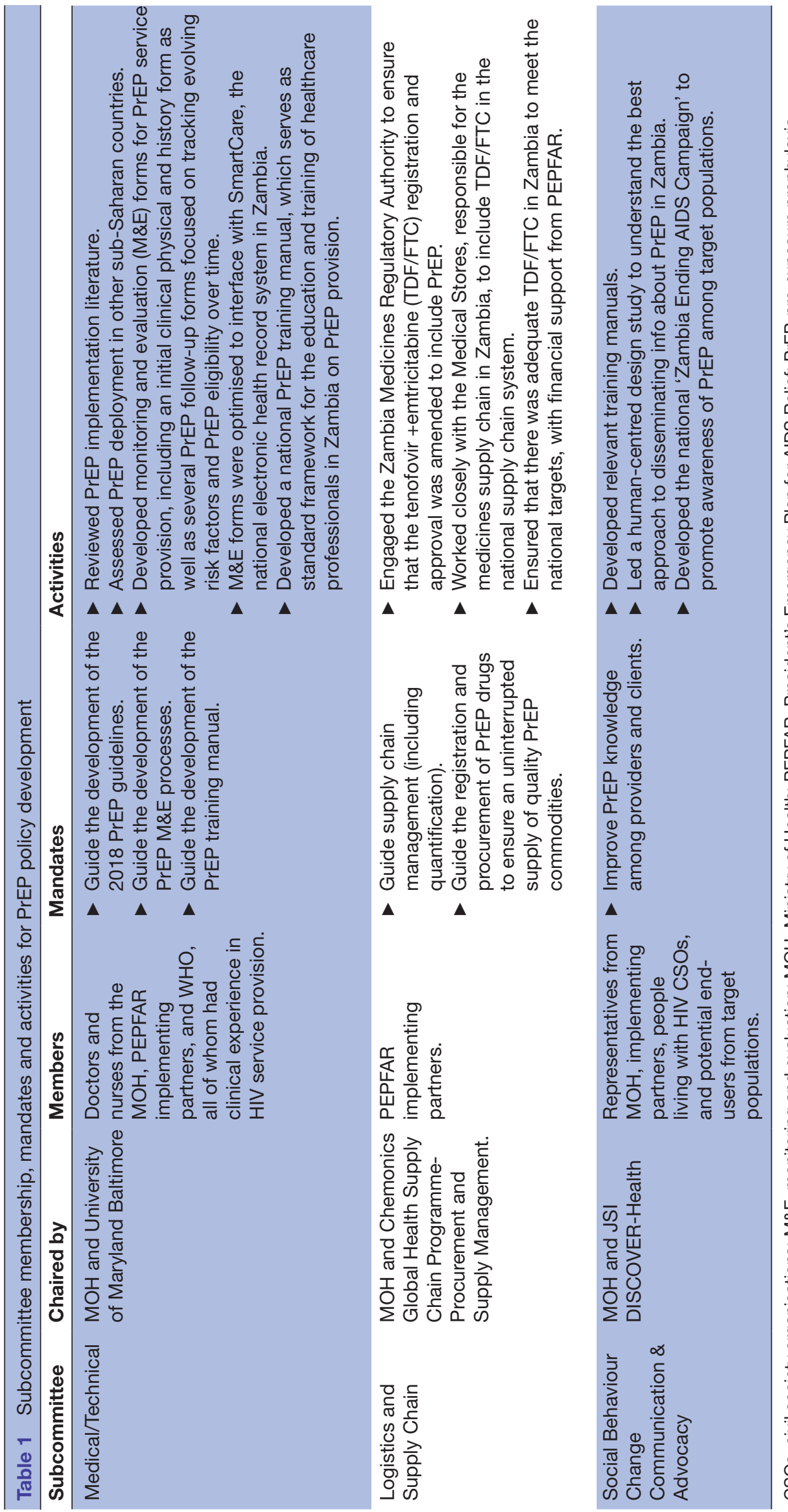




\section{Box 1 Zambia PrEP Risk-based eligibility criteria 2018}

Persons at substantial risk for HIV infection, defined as engaging in one or more of the following activities within the last 6 months:

- Vaginal/anal intercourse without condoms with more than one partner.

- Sexually active with a partner who is known to be HIV-positive or at substantial risk of being HIV-positive.

- Sexually active with an HIV-positive partner who is not on effective treatment (defined as on cART for $<6$ months or not virally suppressed).

- History of STI.

- History of PEP use.

- Sharing injection material or equipment.

cART, combined antiretroviral therapy; PrEP, pre-exposure prophylaxis; PEP, post-exposure prophylaxis; STI, sexually transmitted infection.

Force participated in international workshops to catalyse and share policy development and implementation experiences across southern Africa.

The 2016 Zambian antiretroviral treatment (ART) guidelines recommended PrEP for SDCs and considered for high-risk persons, though risk was not defined. For the 2017 ART guidelines, the Task Force considered recommending PrEP for KPs. However, Zambia remains largely conservative on diverse sexual orientation and gender identity rights. To avoid stigmatising PrEP as a KP intervention and to ensure access to any person at high risk for HIV, the Task Force adopted risk-based criteria for PrEP eligibility, instead of population-specific criteria (box 1).

In addition to the guidelines, $\mathrm{MOH}$ and the Task Force introduced the Implementation Framework \& Guidance for PrEP of HIV Infection in 2018. ${ }^{29}$ This framework provides comprehensive clinical and programme implementation guidance on service delivery of PrEP. This included minimum standards for service delivery with the recommendation to first implement PrEP service delivery in areas of HIV incidence and/or prevalence at existing ART clinics before scaling to community models. Required human resources for PrEP included nurses or clinical officers trained in ART who could provide PrEP, pharmacy staff for dispensation and counsellors and peer educators for education and adherence counselling. The framework employs a people-centred approach to PrEP service delivery, emphasising health coverage, gender equality and health-related rights including accessibility, availability, acceptability and quality. PrEP services are clearly outlined and include guidance and recommendations on:

- Service delivery standards, human resource needs, and supply chain management.

- Regulation of importing, marketing, and control of PrEP medications.

- PrEP indications, usage, drug interactions, side effects and discontinuation.

- Advocacy, education and demand generation materials.

- Recommended social behaviour change strategy.

- Documentation and M\&E of PrEP service delivery.
PrEP pilot and policy implementation and service delivery rollout

Between October 2017 and March 2018, the University of Maryland Baltimore (UMB) and the University Teaching Hospital in Lusaka, with special permission from the $\mathrm{MOH}$, piloted a PrEP intervention package at two public health facilities in Lusaka and Livingstone. The intervention included community education on PrEP alongside training of HCWs on KP sensitivity and PrEP service delivery. Initial results from this pilot demonstrated high levels of interest and uptake among users, but low PrEP continuation by 3 months: among 1002 clients that tested negative for HIV, 326 (33\%) were eligible for PrEP, of whom $116(36 \%)$ elected to begin PrEP; 95 (82\%) of these clients presented for 1-month follow-up and 31 $(27 \%)$ clients for 3-month follow-up. ${ }^{30}$

The lessons learnt from this pilot were crucial to informing the broader national PrEP strategy. Additional training on community awareness and PrEP demand was required to ensure successful programme scale-up. To address this, UMB developed peer-led promotion of PrEP uptake through targeted messaging in KP communities, and also created social media platforms (WhatsApp and Facebook) where current and potential clients could discuss PrEP with mentor users and knowledgeable HCWs. Additionally, the pilot emphasised the need for expanded HCW training and community support for PrEP and KP-friendly services, with services provided in KP safe spaces by HCWs trained to provide services in a non-discriminatory manner. Trainings in PrEP service provision were extended to community health workers (CHWs) and peer supporters who created demand for PrEP and encouraged PrEP continuation. KP peer promoters were recruited from the KP community to disseminate HIV prevention and testing messages with a focus on the benefits of PrEP. Finally, operating hours at PrEP clinics were adjusted to include evenings, and PrEP services were provided closer to target populations in order to mitigate access barriers observed in the pilot study.

The $\mathrm{MOH}$, with financial and technical support from PEPFAR and IPs, began the national roll-out of PrEP in 2018. Activities ranged from policy development, health system strengthening, commodity procurement (laboratory and drug requirements), service delivery (staff, training/mentorship, etc), M\&E and demand creation. Concurrently, in early 2018, the Task Force set targets for national PrEP roll-out, focusing on facilities offering ART in high-burden areas. PEPFAR partners developed and implemented differentiated service delivery (DSD) models to serve the unique needs of each targeted population for early PrEP roll-out. To identify high-risk areas in the community hotspot mapping was conducted. KP safe spaces were set up where PrEP could be provided to MSM and FSWs. Nearby health facilities supported these safe spaces with HCW staff trained in PrEP service delivery as well as KP sensitivity, safety and security. 
For all models of service provision, IPs supported the $\mathrm{MOH}$ to train clinicians, nurses, CHWs, and peer supporters in PrEP service delivery, demand creation, and M\&E.

To aid in public education and demand creation, the Zambia Ending AIDS national HIV prevention campaign was implemented in 2019, with technical support from JSI/USAID DISCOVER-Health. The campaign provided information on HIV prevention with a key focus on PrEP, and disseminated information on PrEP via social media and cellular phone text messages. The campaign was driven by findings from a human-centred design study that found: (1) low awareness of PrEP, including among HCWs, with safety and tolerability concerns; (2) higher awareness among youth and KPs, who viewed PrEP favourably for high-risk situations; (3) AGYW saw PrEP as a way to exercise control over their own HIV prevention, (4) older men viewed PrEP as a way to have safer sex without a condom; (5) young people concerned about parents finding out they were on ARVs; (6) high levels of judgmental and discriminatory attitudes among HCWs.

These findings were used to design the PrEP subcampaign in the broader Zambia Ending AIDS campaign. The key tenets of the campaign include sex positivity (ie, sex without judgement or stigma) and addressing low risk perception; PrEP and combination HIV prevention, as well as a focus on men's health and male-specific health programming; education of HCWs as caring providers of sexual health; and, enhanced health communication tools (figure 2).

\section{PrEP programme M\&E}

In accordance with the national framework M\&E guidance, aggregated PrEP service delivery data including age, sex, KP type and geographical reach are reported semi-annually by IPs into the PEPFAR reporting database (DATIM). The PEPFAR fiscal year (FY) is from October 1 to September 30 each year. To evaluate early PrEP programme scale-up, we analysed deidentified aggregate PrEP service delivery data from DATIM, with ethical clearance granted by ERES, NHRA, UMB and CDC. Data included sex, age, KP type, rates of uptake and follow-up at each clinical visit. We also conducted geo-mapping to overlay provision of PrEP services with population density and HIV prevalence.

\section{Patient and public involvement}

Only aggregate patient data was used in this study. Patient advocates and civil society representatives formed part of the National PrEP Task Force and participated in drafting policy and setting the national policy agenda. Key results about PrEP have been routinely disseminated to the public at national meetings and conferences and other venues.

Figure 2 Selected materials from Zambia ending AIDS campaign. PrEP, pre-exposure prophylaxis. 


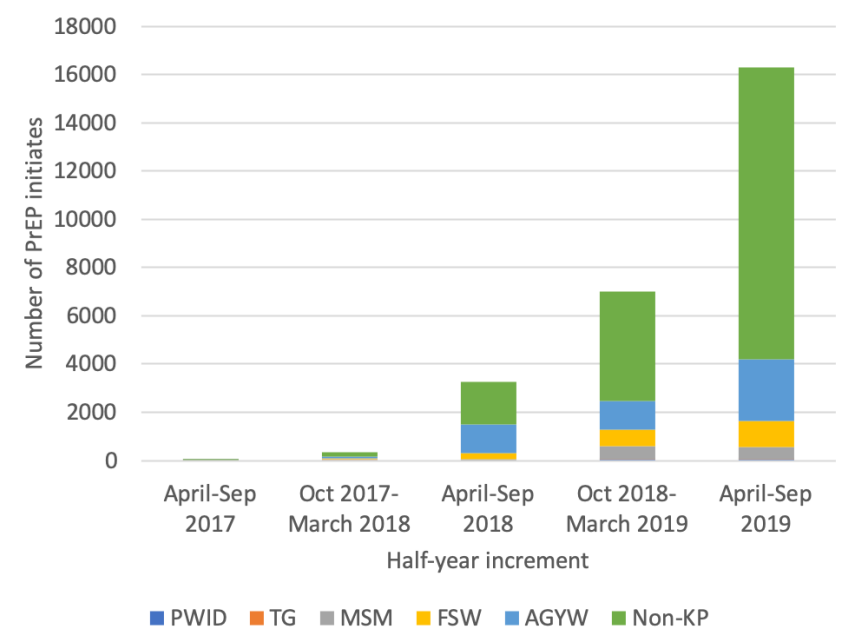

Figure 3 Total number of new PrEP initiates by population type since beginning of Zambian PrEP programme, in halfyear increments. AGYW, adolescent girls and young women; FSW, female sex workers; KP, key population; MSM, men who have sex with men; PWID, people who inject drugs; TG, transgende.

\section{National PrEP scale-up}

\section{Early scale-up}

Between October 2017 and September 2018 (PEPFAR FY 18), a total of 3603 persons initiated PrEP, with particular focus on populations most at risk of contracting HIV, namely AGYW (n=1272, 34\%), FSWs ( $\mathrm{n}=312,9 \%)$ and MSM ( $\mathrm{n}=92,3 \%)$. During this period, nearly half of all PrEP initiates were KPs or priority populations (PPs) (ie, FSW, MSM, TG, PWID, AGYW): $52 \%$ from April to September 2017, 48\% from October 2017 to March 2018, and $46 \%$ from April to September 2018, with the other half being SDCs or general population (figure 3). During this first year of PrEP provision, prior to the national launch, programme scale was limited and IPs largely focused on KPs/PPs.

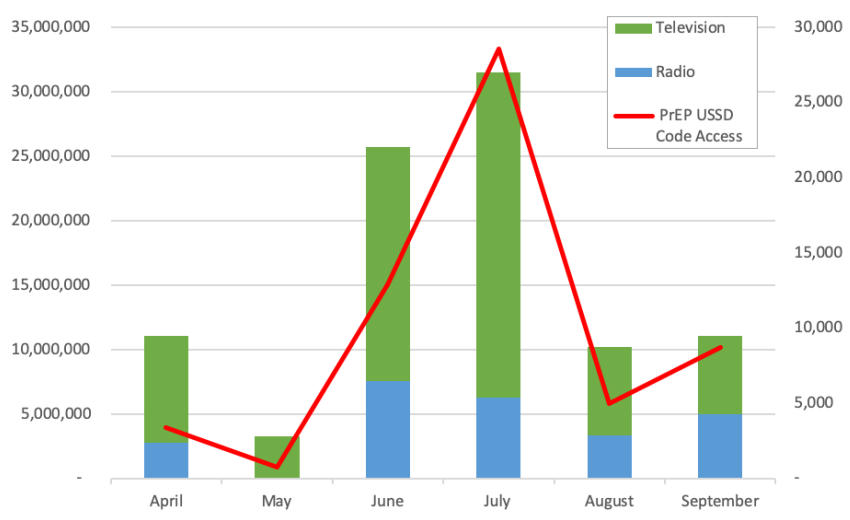

Figure 4 PrEP information access correlates to ending AIDS campaign media broadcasts. Snapshots of national radio listeners and TV broadcasts over a 6-month period in 2019, with overlay of frequency of USSD PrEP code being accessed. USSD PrEP codes track closely with Zambia ending AIDS campaign media broadcasts. PrEP, preexposure prophylaxis.

\section{National HIV prevention campaign}

Between April 2019—when the Zambia Ending AIDS campaign was launched-and September 2019, over 4.5 million people were reached on social media with campaign messaging. PrEP advertisements were broadcast in six major Zambian languages over 90 million times on 22 national and community radio and television stations. From January to September 2019, 140933 people accessed the national toll-free Unstructured Supplementary Service Data (USSD) code for information about PrEP. Of these, 27889 requested information about the nearest PrEP facility. People tended to access the USSD code when there was high rotation of the PrEP adverts on radio, highlighting the importance of sustained PrEP sensitisation (figure 4).

\section{Full scale-up}

By March 2019, access and availability of PrEP had substantially increased, with services available across all ten provinces in 340 sites (of approximately 2000 ARTproviding sites across Zambia). By September 2019, PrEP services delivery had expanded to 728 sites and included health facilities, health outreach posts and non-traditional sites such as PEPFAR DREAMS Centres (community centres for prevention services for AGYW), police camps, prisons, markets and universities. The data did not allow for breakdown of clients served per facility; however, service delivery mapping indicates that PrEP is currently provided in the areas of greatest population density where the HIV burden is most concentrated (figure 5).

Both the HIV prevention campaign and the increase in number of sites offering PrEP has led to a substantial increase in the number of people initiating PrEP. From April-September 2018 to October 2018-March 2019, the number of PrEP initiates more than doubled, from 3248 to 7018 , and this increased an additional two-fold to 16309 new initiates in April-September 2019 (figure 3). By September 2019, a total of 26953 clients had initiated PrEP.

There was also a concurrent increase in KPs accessing PrEP; new FSW initiates increased fourfold (248 to 1062) and MSM initiates increased ninefold (55-504) in the same time period. Interestingly, despite this increase in KPs accessing PrEP, the proportion of KPs as a total of all PrEP users has declined, showing the relative broad uptake and appeal of PrEP among the general population. From April to September 2019, just 26\% of all new PrEP initiates were KPs, a significant decline from $46 \%$ just a year prior. This shift in PrEP user-type may result in continued increased demand for PrEP and PrEP normalisation, and is in line with the aims of the national HIV prevention campaign. This increased proportion of non-KP PrEP users highlights the Zambian PrEP policy's success in not targeting KPs alone, but making PrEP available to any individual at significant risk of acquiring HIV. 

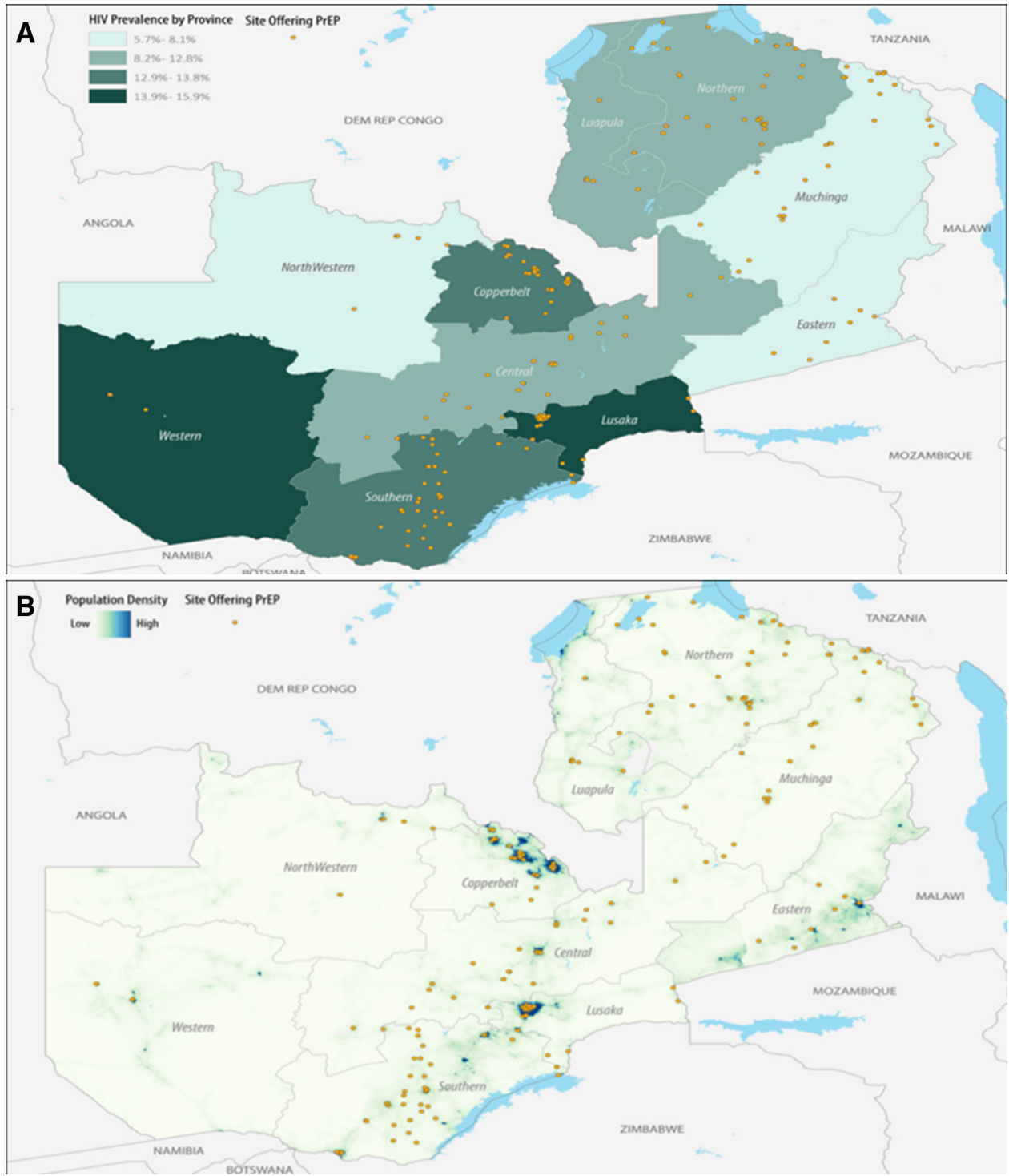

Figure 5 Geographic distribution of HIV PrEP sites in Zambia in 2019. Panel (A) Zambian government health facilities providing PrEP in orange, overlaying HIV prevalence by Province. Panel (B) Zambian government health facilities providing PrEP in orange, overlaying population density by Province. PrEP, pre-exposure prophylaxis.

\section{PrEP continuation}

Continuation data are not consistently collected and reported in the national system or in DATIM. However, in the UMB pilot study, among 116 clients who enrolled on PrEP, 95 (82\%) returned at 1 month and $31(27 \%)$ returned at 3 months. PrEP continuation at 3 months was highest for SDCs (42\%), then TG (33\%), then MSM $(23 \%)$ and lowest among FSWs $(21 \%){ }^{30}$

JSI conducted a snapshot analysis of data at their supported sites for persons initiated on PrEP from January 2018 to April 2020, whereby only those on PrEP for a certain duration of time were included in the corresponding denominator. In this analysis, 42\% (6358/15 110) of persons who had been initiated on PrEP returned at 1 month for PrEP refills. Persistence at 3 months was $35 \%$ (4709/13 383), 6 months 25\% (2507/10 199), 9 months $13 \%(980 / 7413)$ and by 12 months was only $11 \%$ $(570 / 5246)$.

\section{DISCUSSION}

Between 2016 and 2018, Zambia developed health policy and a national framework to support the introduction and national scale-up of PrEP to those most at risk of HIV infection. In the first year, initial guidelines were developed and pilot implementations were conducted, serving less than 4000 people. In year two, more expansive, riskbased criteria were adopted, and scale-up led to a sixfold increase with over 23000 persons starting PrEP. The introduction of PrEP is reflective of a collaborative effort from a multidisciplinary task force comprised of the Zambian government, PEPFAR, WHO, UNAIDS, PEPFAR IPs and CSOs with the mandate to rapidly develop the national strategy and improve access and delivery of PrEP to at-risk groups. Mapping of PrEP services to HIV prevalence demonstrates that PrEP services are largely being provided where there is greatest need. Provision of DSD models such as PrEP service delivery in community safe 
spaces was found to support PrEP uptake among KPs. ${ }^{31}$ Further support by IPs is needed to ensure that PrEP is available to all populations at substantial risk of HIV, including those in rural areas. Further research and innovative service delivery models are needed to address barriers to access and continuation of PrEP services for those who continue to be at risk of HIV infection.

While Zambia was able to achieve rapid roll-out and implementation of PrEP, estimates of continuation on PrEP remains low. It should be noted that these estimates are partner-specific and more targeted studies are needed. However, when compared with other real-world implementations in Africa, 25\% continuation at 6 months and $11 \%$ at 12 months is in line with other studies: the Kenya demo project found 6 months continuation rates of $15 \%$ among MSM, 14\% among FSWs, and 10\% among women ${ }^{32}$ while the TAPS study found $30 \%$ continuation at 6 months among South African FSWs. ${ }^{17}$

Further understanding the drivers of PrEP discontinuation is needed to improve uptake and continuation of PrEP for those at continued risk of HIV infection, as well as ongoing community education and demand generation. Avenues to reduce PrEP stigma and discrimination should be explored, such as using different branding for PrEP drugs versus ARVs. For PrEP to reach its full potential it needs to be available at all primary health facilities and through DSD models. Finally, augmenting advocacy efforts may help increase PrEP access in contexts where KPs are largely stigmatised.

Limitations of this study include the reliance on aggregate data and the lack of patient perspective. Patient-level analyses are needed to understand who stays on PrEP and who does not, while qualitative studies are needed to illuminate why certain people take up PrEP while others decline, as well as reasons for adherence or discontinuation. Further study in these areas is critical to ensure that PrEP is provided to those who can most benefit from it.

The PrEP roll-out in Zambia can be compared with the successful PrEP scale up effort in Kenya. ${ }^{33}$ Similar to Zambia, Kenya established a PrEP Technical Working Group; however they opted for six thematic subcommittees instead of three. Kenya also achieved quick roll-out, from launching guidelines in July 2016 to launching national PrEP scale-up in May 2017. Kenya similarly recognised the strategic importance of partnerships among government, IPs, and CSOs. They also highlighted the need for training of HCWs, the importance of demand creation and social marketing, community-led programmes, PrEP integration into commodity management systems, and the importance of engaging with $\mathrm{MOH}$ from the outset.

A unique element of Zambia's PrEP scale-up was the decision to use risk-based criteria as opposed to population-based criteria. It has been argued that national PrEP programmes often follow a certain sequence, the 'first wave' being PrEP delivery for KPs followed by a 'second wave' for PPs, especially AGYW. ${ }^{34}$ By eliminating $\mathrm{KP} / \mathrm{PP}$-typology from the eligibility criteria, we ensure that all persons at risk for HIV can access PrEP, regardless of how they identify. This is critical in the setting of a generalised epidemic such as Zambia, where multiple groups of persons are at ongoing risk. Given that certain KPs are at high risk of stigmatisation, by not associating the PrEP programme with KPs we avoided issues around PrEP being used as a marker of certain activities.

However, a potential drawback of this approach is that it risks not reaching groups most at risk. Indeed initial uptake of PrEP in Zambia included a high proportion of general population (including SDCs) compared with KPs and PPs. This mirrors the uptake in both Kenya ${ }^{33}$ and Uganda ${ }^{35}$ both of which saw over $60 \%$ of PrEP being taken up by general population and SDCs. This may be due to broader inclusion criteria which may also be related to low continuation rate as we may not have reached the most at-risk persons who are likely to continue. This type of broad approach may also reduce overall cost-effectiveness of the PrEP programme more generally. However, though Zambia employed a risk-based approach, there was substantial investment in KP-specific programming to ensure uptake by those most at risk of infection.

Another advantage of a risk-based approach is that it allows for evaluation of 'seasons of risk' with persons starting and stopping PrEP based on an objective assessment of their risk at any point in time. This data can also be used to compare different strategies for PrEP roll-out, using our recently developed programme evaluation metric, which allows for comparison of PrEP programmes serving KPs and PPs. ${ }^{36}$

\section{Lessons learnt}

We present an example of health policy development that led to rapid national implementation and service delivery scale-up. Key elements of success included a firm grounding in evidence-based approaches which enabled a strong case for PrEP implementation; a multisector, interdisciplinary task force which allowed for rapid advancement of policy and implementation; and a well-planned implementation pilot, which provided key lessons that informed the national scale-up. A particular strength of Zambia's PrEP guidelines is the risk-based approach, making it an intervention available to all persons at risk, not just members of certain populations. Conducting a client-centred public awareness campaign is key to disseminating knowledge about PrEP and driving demand creation. Further detailed quantitative and qualitative research is needed to understand drivers of uptake, continuation and discontinuation. Such approaches to PrEP health policy development and service delivery implementation can serve as a model to other countries seeking to implement PrEP for HIV prevention.

\section{Author affiliations}

${ }^{1}$ Center for International Health, Education, and Biosecurity, University of Maryland School of Medicine, Baltimore, Maryland, USA

${ }^{2}$ Adult Infectious Diseases Center, University Teaching Hospital, Lusaka, Zambia ${ }^{3}$ Division of Infectious Diseases, Department of Medicine, University of Zambia School of Medicine, Lusaka, Zambia 
${ }^{4}$ National HIV/AIDS/STI/TB Council, Lusaka, Zambia

${ }^{5}$ Ministry of Health, Lusaka, Zambia

${ }^{6}$ John Snow International, Lusaka, Zambia

${ }^{7}$ Chemonics Global Health Supply Chain Program-Procurement and Supply

Management, Lusaka, Zambia

${ }^{8}$ Ciheb Zambia, Lusaka, Zambia

${ }^{9}$ Department of Global Health, Boston University School of Public Health, Boston,

Massachusetts, USA

${ }^{10}$ Health Economics and Epidemiology Research Office, Wits University,

Johannesburg-Braamfontein, South Africa

${ }^{11}$ World Health Organization, Lusaka, Zambia

${ }^{12}$ U.S. Centers for Disease Control and Prevention, Lusaka, Zambia

${ }^{13}$ U.S. Agency for International Development, Lusaka, Zambia

Acknowledgements The authors gratefully acknowledge the health care workers, community health workers, client advocates, and clients who made this work possible.

Contributors All authors contributed to PrEP health policy and development as participants in the Zambia National PrEP Task Force meetings. CC and DM designed the manuscript concept, and led the writing and coordination, with assistance from MN. CC, DM, MN, DN, LKM, MM, EM, LBM, TC, BN, CH, LC, JO and HO all contributed by writing and editing pertinent sections. $\mathrm{CC}, \mathrm{HO}, \mathrm{BN}, \mathrm{CH}$ and $\mathrm{MN}$ aided in data analysis and results reporting. All authors aided in reviewing and editing the paper.

Funding This work was supported by multiple donors and funding agreements. Key contributions include the US President's Emergency Plan for AIDS Relief (PEPFAR) via the CDC-funded CIRKUITS (6NU2GGH002123-02-07) and Z-CHECK (5 NU2GGH001913-05-00) and USAID-funded DISCOVER-Health (AID-611-A-16-00004) projects. PEPFAR attribution of support: This publication has been supported by the US President's Emergency Plan for AIDS Relief (PEPFAR) through the US Centers for Disease Control and Prevention (CDC).

Disclaimer The findings and conclusions in this publication are those of the authors and do not necessarily represent the official position of the funding agencies.

Map disclaimer The depiction of boundaries on this map does not imply the expression of any opinion whatsoever on the part of BMJ (or any member of its group) concerning the legal status of any country, territory, jurisdiction or area or of its authorities. This map is provided without any warranty of any kind, either express or implied.

Competing interests None declared.

Patient and public involvement Patients and/or the public were involved in the design, or conduct, or reporting, or dissemination plans of this research. Refer to the Methods section for further details.

Patient consent for publication Not required.

Ethics approval Ethical approval for this retrospective analysis of aggregate routine program data was covered by the routine PEPFAR MER data protocol approved by the following Institutional Review Boards (IRBs): 1. ERES Converge IRB, Lusaka, Zambia—Ref. No. 2020-Mar-015. 2. University of Maryland Baltimore, Maryland-Ref. No. HP- 00086064. 3. National Health Research Authority, Lusaka, Zambia-IRB waiver granted. 4. US Centers for Disease Control and Prevention-non-research determination granted, Ref. No. CGH HSE 2017-519.

Provenance and peer review Not commissioned; externally peer reviewed.

Data availability statement Data are available on reasonable request. The data used in this study, and additional data, may be made available by emailing hobra@ usaid.gov.

Open access This is an open access article distributed in accordance with the Creative Commons Attribution Non Commercial (CC BY-NC 4.0) license, which permits others to distribute, remix, adapt, build upon this work non-commercially, and license their derivative works on different terms, provided the original work is properly cited, appropriate credit is given, any changes made indicated, and the use is non-commercial. See: http://creativecommons.org/licenses/by-nc/4.0/.

\section{ORCID iDs}

Cassidy W. Claassen http://orcid.org/0000-0001-5059-7894

Brooke E. Nichols http://orcid.org/0000-0003-4682-4999

Cheryl Hendrickson http://orcid.org/0000-0002-
REFERENCES

1 WHO. Guideline on when to start antiretroviral therapy and on preexposure prophylaxis for HIV, 2015.

2 Baeten JM, Donnell D, Ndase P, et al. Antiretroviral prophylaxis for HIV prevention in heterosexual men and women. N Engl J Med 2012;367:399-410.

3 Grant RM, Lama JR, Anderson PL, et al. Preexposure chemoprophylaxis for HIV prevention in men who have sex with men. N Engl J Med 2010;363:2587-99.

4 Thigpen MC, Kebaabetswe PM, Paxton LA, et al. Antiretroviral preexposure prophylaxis for heterosexual HIV transmission in Botswana. N Engl J Med 2012;367:423-34.

5 Van Damme L, Corneli A, Ahmed K, et al. Preexposure prophylaxis for HIV infection among African women. N Engl J Med 2012;367:411-22.

6 Marrazzo JM, Ramjee G, Richardson BA, et al. Tenofovir-based preexposure prophylaxis for HIV infection among African women. $N$ Engl J Med 2015;372:509-18.

7 Fonner VA, Dalglish SL, Kennedy CE, et al. Effectiveness and safety of oral HIV preexposure prophylaxis for all populations. AIDS 2016;30:1973-83.

8 Bailey JL, Molino ST, Vega AD, et al. A review of HIV pre-exposure prophylaxis: the female perspective. Infect Dis Ther 2017;6:363-82.

9 Valente PK, Mantell JE, Masvawure TB, et al. "I Couldn't Afford to Resist": Condom Negotiations Between Male Sex Workers and Male Clients in Mombasa, Kenya. AIDS Behav 2020;24:925-37.

10 Irungu EM, Baeten JM. PrEP rollout in Africa: status and opportunity. Nat Med 2020;26:655-64.

11 Baeten JM, Heffron R, Kidoguchi L, et al. Integrated delivery of antiretroviral treatment and pre-exposure prophylaxis to HIV-1Serodiscordant couples: a prospective implementation study in Kenya and Uganda. PLoS Med 2016;13:e1002099.

12 Heffron R, Ngure K, Odoyo J, et al. Pre-exposure prophylaxis for HIV-negative persons with partners living with HIV: uptake, use, and effectiveness in an open-label demonstration project in East Africa. Gates Open Res 2017;1:3.

13 Bekker L-G, Roux S, Sebastien E, et al. Daily and non-daily preexposure prophylaxis in African women (HPTN 067/ADAPT Cape town trial): a randomised, open-label, phase 2 trial. Lancet HIV 2018;5:e68-78.

14 Henderson F, Taylor A, Chirwa L. Characteristics and oral PrEP adherence in the TDF2 open-label extension in Botswana. J Int AIDS Soc 2015;18.

15 Cowan FM, Davey C, Fearon E, et al. Targeted combination prevention to support female sex workers in Zimbabwe accessing and adhering to antiretrovirals for treatment and prevention of HIV (SAPPH-IRe): a cluster-randomised trial. Lancet HIV 2018;5:e417-26.

16 Mboup A, Béhanzin L, Guédou FA, et al. Early antiretroviral therapy and daily pre-exposure prophylaxis for HIV prevention among female sex workers in Cotonou, Benin: a prospective observational demonstration study. J Int AIDS Soc 2018;21:e25208.

17 Eakle R, Gomez GB, Naicker N, et al. HIV pre-exposure prophylaxis and early antiretroviral treatment among female sex workers in South Africa: results from a prospective observational demonstration project. PLoS Med 2017;14:e1002444.

18 Pillay D, Stankevitz K, Lanham M, et al. Factors influencing uptake, continuation, and discontinuation of oral PrEP among clients at sex worker and MSM facilities in South Africa. PLoS One 2020;15:e0228620.

19 Wahome EW, Graham SM, Thiong'o AN, et al. PrEP uptake and adherence in relation to HIV-1 incidence among Kenyan men who have sex with men. EClinicalMedicine 2020;26:100541.

20 Zambian Ministry of Health, Centers for Disease Control and Prevention, ICAP Columbia University, Central Statistics Office [Zambia]. Zambia population-based HIV impact assessment (ZamPHIA): final report. Lusaka, Zambia, 2016.

21 Smith AD, Tapsoba P, Peshu N, et al. Men who have sex with men and HIV/AIDS in sub-Saharan Africa. Lancet 2009;374:416-22.

22 Poteat T, Scheim A, Xavier J, et al. Global epidemiology of HIV infection and related Syndemics affecting transgender people. $J$ Acquir Immune Defic Syndr 2016;72(Suppl 3):S210-9.

23 Dobra A, Bärnighausen T, Vandormael A, et al. Space-time migration patterns and risk of HIV acquisition in rural South Africa. AIDS 2017;31:137-45.

24 Heffron R, Chao A, Mwinga A, et al. High prevalent and incident HIV-1 and herpes simplex virus 2 infection among male migrant and non-migrant sugar farm workers in Zambia. Sex Transm Infect 2011;87:283-8.

25 Henostroza G, Topp SM, Hatwiinda S, et al. The high burden of tuberculosis (TB) and human immunodeficiency virus (HIV) in a large Zambian prison: a public health alert. PLoS One 2013;8:e67338. 
26 Telisinghe L, Charalambous S, Topp SM, et al. HIV and tuberculosis in prisons in sub-Saharan Africa. Lancet 2016;388:1215-27.

27 Ministry of Health Zambia. Zambia consolidated guidelines for prevention and treatment of HIV infection. Lusaka, Zambia: Ministry of Health, 2016.

28 Ministry of Health Zambia. Zambia consolidated guidelines for prevention and treatment of HIV infection. Lusaka, Zambia: Ministry of Health, 2018.

29 Ministry of Health Zambia. Implementation Framework \& Guidance for Pre-Exposure Prophylaxis of HIV Infection. Lusaka, Zambia: Ministry of Health, 2018.

30 Claassen CW ML, Lavoie MC, Gashongore I. Pre-Exposure prophylaxis in Zambia: policy engagement and initial implementation. AIDS 2018 Amsterdam, Netherlands 2018.

31 Mwango L, Kafunda I, Lubinda R. Prep initiation in community safe spaces increases PrEP access among key and priority populations in Zambia. Accepted to International AIDS Society Conference Berlin, Germany, 2021.
32 Kyongo JK KM, Karugu FR, Ochieng C. How long will they take it? Oral preexposure prophylaxis (PrEP) retention for female sex workers, men who have sex with men and young women in a demonstration project in Kenya. 22nd International AIDS Conference, Amsterdam, 2018.

33 Masyuko S, Mukui I, Njathi O, et al. Pre-exposure prophylaxis rollout in a national public sector program: the Kenyan case study. Sex Health 2018;15:578-86.

34 Eakle R, Venter F, Rees H. Pre-exposure prophylaxis (PrEP) in an era of stalled HIV prevention: can it change the game? Retrovirology 2018;15:29.

35 Acom PKL, Bwanika J. Mobile health approaches with facility-based care to improve access and adherence to pre-exposure prophylaxis (PrEP) in Lango region, Uganda. Reviews in Antiviral Therapy \& Infectious Diseases 2020;10.

36 Hendrickson C, Long L, van de Vijver D, et al. Novel metric for evaluating pre-exposure prophylaxis programme effectiveness in real-world settings. Lancet HIV 2020;7:e294-300. 